\title{
An Effective Image Coding Method using Lattice Vector Quantization in Wavelet Domain
}

\author{
Lizhi Zhang ${ }^{1}$, Mingrui Zhang ${ }^{2}$, Qinghe Pan $^{1}$, Tao Wang ${ }^{3}$, \\ Zhijie Zhao ${ }^{1}$ and Xuesong Jin ${ }^{1}$ \\ ${ }^{1}$ School of Computer and Information Engineering, Harbin University of Commerce, \\ Harbin, 150028, China \\ ${ }^{2}$ College of Science, Harbin Engineering University, Harbin, 150001, China \\ ${ }^{3}$ Network and Educational Technology Center, Harbin University of Commerce, \\ Harbin, 150028, China \\ zhanglizhi58@163.com,471299608@qq.com,570749130@qq.com, \\ wangtaohsd@126.com,zhijiezzz@yahoo.com.cn,jinxuesong@163.com
}

\begin{abstract}
As the digital information must be stored and recovered in an effective ways, it is necessary to compress data in image coding, wavelet transform and lattice vector quantization are adopted in this paper to improve the compression effective of image coding. We first propose the two dimensional spatial wavelet decomposition of image, and then we present lattice Vector Quantization coding scheme for each image subband, lattice vector quantization can make full use of the correlation between the image wavelet coefficients to remove the information redundancy, and the coding method of lattice quantization can be effective because of applying the symmetries of the lattice. The establishment of rate distortion (RD) model suitable for lattice vector quantization of wavelet image coder is also important for image compression, study shows that the RD performance for the spatial subbands are fitted by an exponential form theoretical model, this yields an analytical solution to the bit rate distribution issue, we explore an effective rate control scheme by using the lagrangian optimization method to distribute the bit rate for the spatial subbands. The experimental results show that the proposed algorithm can achieve better compressing effect with minimum loss.
\end{abstract}

Keywords: Lattice Vector Quantization (LVQ); Discrete Wavelet Transform (DWT); image coding; rate distortion function; rate allocation

\section{Introduction}

With the increasing development of technology, the application of digital images becomes more and more popular. As the digital information must be stored and recovered in an effective ways, it is necessary to compress data in image coding, actually the image is a kind of redundant data, and it is possible to delete some of the redundant data included in images by using data compressing methods. The original image can be transformed in order to remove some of the dependency among pixels, then only the few significance coefficients need to be encoded, and the study shows that the image coding system using wavelet transform can achieve better performance than the system using discrete cosine transform 
(DCT), it can not only achieve good performance of compressing, but also can achieve effective scalability [1].

After decomposing the original image, the wavelet coefficients can be quantized by scalar quantization (SQ) as in JPEG2000 standards or vector quantization [2]. VQ that encodes sequences of outputs would provide some improvement in terms of performance over scalar quantization. It first creates a representative group of sequences, and for a given sequence, vector quantization would then represent the given output sequence with one of the representatives of the representative group. The vector quantization technology has been originated in 1970s, and today, as an efficient data compression technology, it is widely used in some areas such as audio coding, image coding, video coding, and pattern recognition etc., [3-6].

Rate control is the basic problem for the image coding system. It solves the issue to determine the encoding parameters so that the decoded image quality is best under the constraint of certain fixed bit rate. So it attracts more study interest in recent years, for instance, Wang Qi[7] et al., focused their research attention on the enhancement layer rate distribution issue of the progressive fine granular scalable video coding system. Vidhya Seran [8] et al., focused their research attention on the rate distribution issue to overcome the fluctuation of distortion in the wavelet based video encoders. In this paper, we explore the rate control method for wavelet transformed vector quantization image coding system, we first propose the two dimensional wavelet transformed vector quantization image encoder. Then we propose the RD function of each subband. After analyzing the rate control issue, a rate control scheme for two dimensional wavelet transformed image coder is proposed by the Lagrangian optimization algorithm. Finally, the experimental results and analyses are presented.

\section{Wavelet Decomposition of Image}

Wavelet becomes popular approach to decompose a signal recent years, the wavelet transform is a decomposition that represents signal $f(t)$ in shifted and dilated versions of special selected wavelet function $\psi(t)$, and the functions:

$$
\psi_{j, k}(t)=2^{-j / 2} \psi\left(2^{-j} t-k\right), \quad j, k \in Z
$$

Forms an orthonormal base, and signal $f(t)$ can be decomposed as the following equation [9]:

$$
f(t)=\sum_{j, k \in Z} w_{j, k} \psi_{j, k}(t), w_{j, k}=\int f(t) \psi_{j, k}^{*}(t) d t
$$

One of the most popular wavelet transform applications is image process, for example, the JPEG 2000 standard uses wavelets to decompose the image. Images are two dimensional signals, and the decomposition procedure can be illustrated as Figure 1. The Discrete Wavelet Transform (DWT) performs the decomposition of an image into multiple resolution representation, and the discrete wavelet transform of an image can be illustrated in Figure 2. The spatial subbands LL1, LH1, HL1 and HH1 can be obtained after two dimensional wavelet decomposition of the image. The first subband represents the main image information, and the other three subbands represent the additional image information in the horizontal, diagonal, and vertical directions. The subbands LL2, LH2, HL2 and HH2 can be achieved after a further two dimensional 
wavelet decomposition of LL1. If decomposing LL2 further, a growing quad tree can be obtained as illustrated in Figure 3 [10].

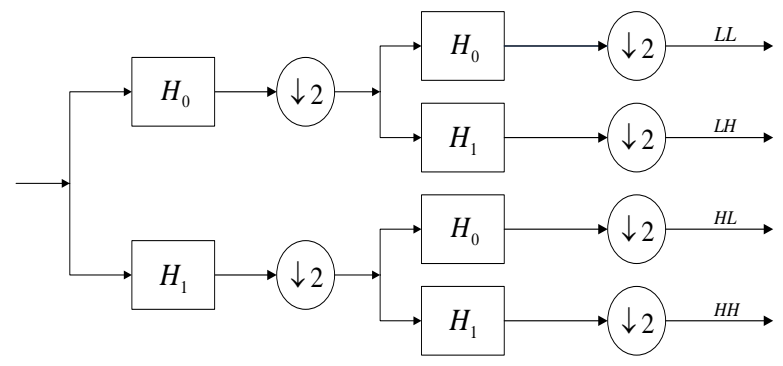

Figure 1. Subband Decomposition of an Image

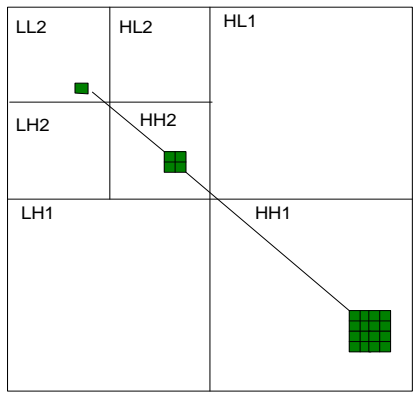

Figure 2. The Image Wavelet Decomposition Subbands

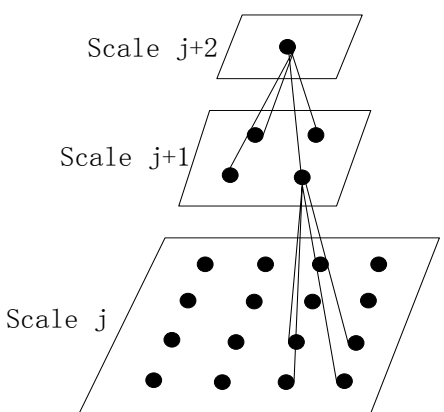

Figure 3. The Quad Trees of Wavelet Coefficient

\section{Wavelet Based Image Coding using Lattice Vector Quantization}

\subsection{Wavelet Based Image Coding with Vector Quantization}

For wavelet based image coding system using vector quantization, we obtain the wavelet coefficients of the image after the wavelet decomposition on the original image. Then it groups the wavelet coefficients into vectors, the vector forms the input to the vector quantizer. There is a group of vectors named codebook at both the encoder and decoder of the vector quantizer. The vectors in the codebook are called codeword, each codeword is allocated an index. The encoder compare the input vector with every codeword in the codebook to search for the codeword which closest to the input vector, the codeword index is sent to the decoder. As the decoder has the same codebook, it replaces index with associated codeword, the components of this codeword are 
quantized values of the wavelet coefficients, then inverse wavelet transform and reconstructed the image, the entire coder can be illustrated in Fgure 4. And the decoder is the inverse of this process.

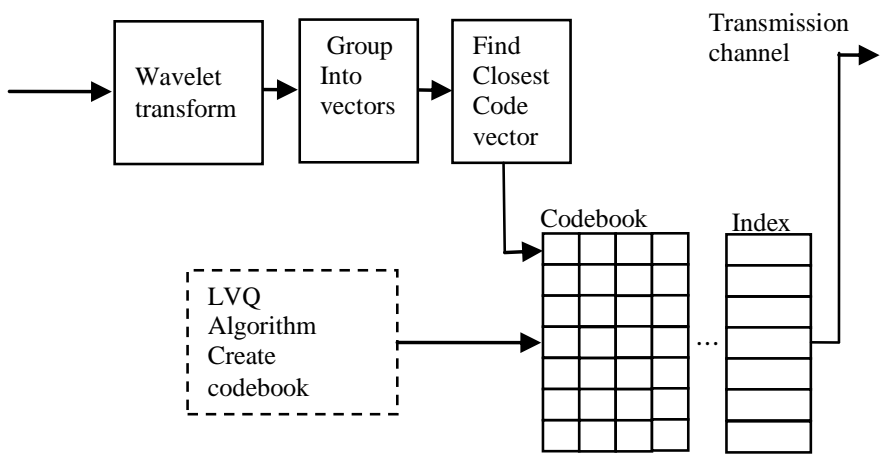

Figure 4. The Wavelet based Vector Quantization Image Coder

\subsection{Lattice Vector Quantization}

Compared with full search algorithms such as LBG algorithm, lattice quantizer can reduce computational complexity due to its regular structure. A lattice is a set of regularly arranged vectors, and can be mathematically defined as follows [11]:

$$
\Lambda=\left\{\mathrm{x}: \mathrm{x}=\sum_{i=1}^{L} u_{i} a_{i}\right\}
$$

Where $\left\{a_{1}, a_{2}, \ldots, a_{L}\right\}$ are $L$ independent $L$ dimensional vectors, and the $u_{i}$ are integers, Figure 5 shows an example of lattice in two dimensions.

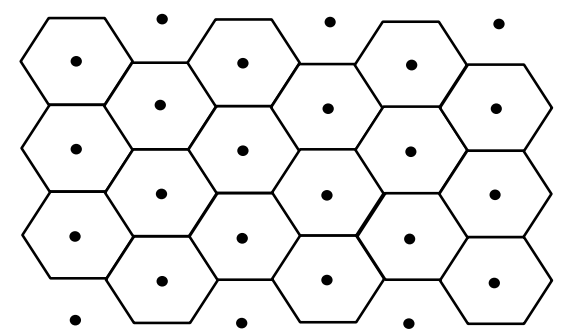

Figure 5. An Example of Lattice in Two Dimensions

\subsection{Lattice Vector Quantization Codebook Design}

Indexing code vector is important for lattice quantization, it need a tradeoff between storage requirements and computational complexity. The indexing method of lattice quantization can be effective when applying the symmetries of the lattice [12]. A lattice is a set of regularly arranged vectors, each vector is located on a constant energy surface, and the surface is with constant radius. So a prefix code can be allocated to each vector, where the prefix encodes the radius of the vector located and the suffix encodes the position it located on the respective surface [13]. The bit rate cost of the 
prefix depends on the number of the surfaces, and the bit rate cost of the suffix depends on the population of the constant radius surface which the vector located.

\section{RD Performance for Single Subband}

The basic for the rate control issue is the rate distortion (RD) function. We first perform the bit rate computation by empirical histogram method so as to establish the $\mathrm{RD}$ model for a single subband. Then the distortion caused by quantization errors is analyzed. Based on these, we propose an exponential form rate distortion model for the spatial subbands.

\subsection{Bit Rate Estimation for Single Subband}

We use the histogram method to compute the mutual information between the random variables related to inter and intra scale wavelet coefficients expressed as $X$ and $Y$, the random variables $X$ and $Y$ are first partitioned into intervals with certain resolution, and then we calculate the joint and marginal probabilities. Finally the mutual information can be computed by using the formula [14], and it is the limit that the bit rate compression could be close to.

$$
\begin{gathered}
I(X ; Y)=E_{X Y}\left[\log \frac{p(x, y)}{P(x) P(y)}\right] \\
I(X ; Y)=D(P(x, y) \| P(x) P(y))
\end{gathered}
$$

Where $D(. \|$.$) denotes Kullback-Leibler divergence between the two distributions,$ also called as the relative entropy.

\subsection{The Distortion Estimation for a Single Subband}

The quantization errors result in distortion in wavelet based image coder, and we often use deadzone uniform quantizer in image coders. Assuming that the quantization step is $\Delta$, then the coefficients within $(-\Delta, \Delta)$ are quantized to zero, and also be named insignificant coefficients, however, the coefficients greater than $\Delta$ are named significant coefficients. So the total distortion contains two parts, one part is the distortion due to discarding the insignificant coefficients, and the other part is the distortion due to quantizing the significant coefficients, the MSE distortion D caused by quantization errors in wavelet-based image coder can be computed by the following formula [15].

$$
D=\frac{1}{N} \sum_{|x|<\Delta}|x|^{2}+\frac{M}{N} \frac{\Delta^{2}}{12}
$$

Where $N$ is the total number of coefficients $x$, and $\mathrm{M}$ is the total number of significant coefficients.

\subsection{RD Performance for a Single Subband}

Before investigating an effective rate control scheme, the RD performance for the spatial subband need to be first explored. Based on the calculation of bit rate and distortion mentioned above, we can approximately model the rate distortion performance of the spatial subband by the exponential form function as below. 


$$
D=\sigma^{2} e^{-\alpha R}
$$

Where $\alpha$ denotes the parameter of coding efficiency, and $\sigma$ denotes the wavelet coefficients variance. We also carry out the experiments to verify the RD performance for the spatial subband, Lena $(256 * 256 * 8$ bits) test image is chosen, and a $9 / 7$ filter with a three level spatial decomposition is used to calculate the wavelet coefficients. Figure 6 shows the coding distortion versus the bit rate in case of LL3, LH3, HL2 and HH1 subbands for the test image. The experiments show that the curves of RD performance for the spatial subbands are fitted by the theoretical model with the exponential form as equation (7).
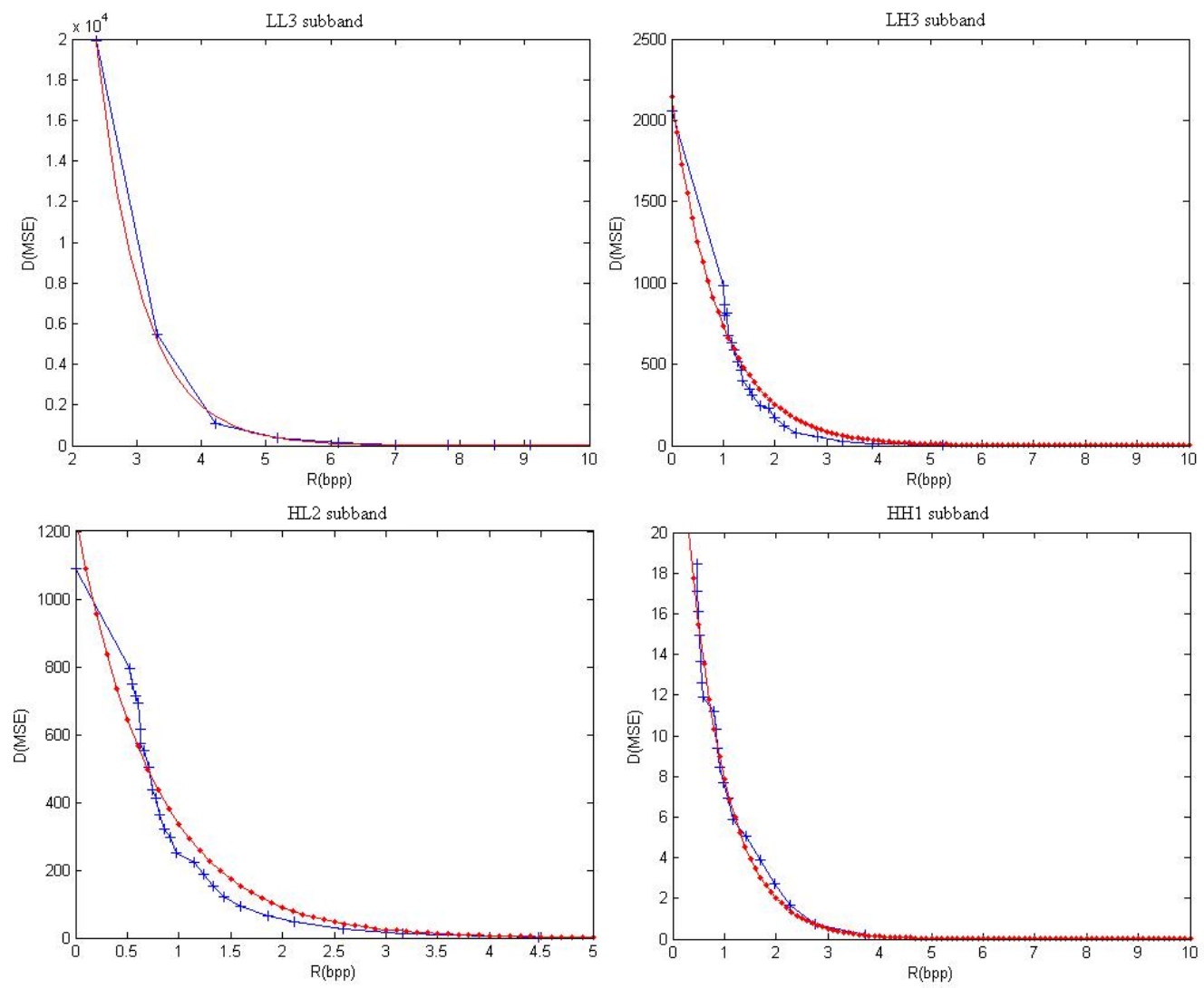

Figure 6. The RD Performance and its Theoretical Model Verification for Subbands of Lena Image, Top Left: LL3 Subband; Top Right: LH3 Subband; Bottom Left: HL2 Subband; Bottom Right: HH1 Subband

\section{Rate Control for the Image Coder}

\subsection{The Description of Rate Control Problem}

The rate control scheme is to determine the coding parameters so that the decoded reconstruction image quality is best under the constraint of a certain fixed bit rate. In spatial filtering structure with $T$ levels there are $3 T+1$ subbands in an image. Let $N$ is the number of subbands of an image, for instance, $N=10$ when taking 3 level 
decomposition of an image. Let $R$ is the bit rate allocated to the image. The rate control issue can be expressed as the following formula, i.e., given the bit rate $R$.

$$
R_{1}+R_{2}+R_{3}+\ldots+R_{N}=R \min \left(D_{1}+D_{2}+D_{3}+\ldots+D_{N}\right)
$$

Where $R_{i}$ and $D_{i}, i=1,2, \ldots, N$, denote the coding bit rate and the distortion for each subband, correspondingly. The aim of the proposed rate control method is to assign the bit rate so that the total distortion is minimized.

\subsection{The Proposed Rate Control Algorithm}

The rate control issue can be dealt with by using Lagrangian optimization algorithm. The issue is to minimize the distortion of the image coder under the constraint of a total bit rate:

$$
R=\sum_{i=1}^{N} \mathrm{R}_{i}
$$

i.e., to minimize

$$
\Phi\left(R_{1}, R_{2}, \ldots, R_{N}\right)=\sum_{i=1}^{N} D_{i}+\lambda\left(\sum_{i=1}^{N} R_{i}-R\right)
$$

Where $\lambda$ denotes a constant which will be determined, in order to minimize (10), we have

$$
\frac{\partial \Phi}{\partial R_{i}}=0, \quad i=1,2, \ldots, N
$$

which yields

$$
R_{i}=\frac{M_{i}}{\alpha_{i}}\left(\ln \sigma_{i}^{2} \alpha_{i}-\ln \lambda\right)
$$

Where $M_{i}$ is the ratio of the number of pixels in subband $i$ to the number of pixels in the whole image, and

$$
\ln \lambda=\frac{\sum_{i=1}^{N} \frac{M_{i}}{\alpha_{i}} \ln \sigma_{i}^{2} \alpha_{i}-R}{\sum_{i=1}^{N} \frac{M_{j}}{\alpha_{i}}}
$$

Finally, the allocated rate for each subband is:

$$
R_{i}=\frac{M_{i}}{\alpha_{i}}\left(\ln \sigma_{i}{ }^{2} \alpha_{i}-\frac{\sum_{j=1}^{N} \frac{M_{j}}{\alpha_{j}} \ln \sigma_{j}{ }^{2} \alpha_{j}-R}{\sum_{j=1}^{N} \frac{M_{j}}{\alpha_{j}}}\right), i=1,2, \ldots, N
$$

Note that, we need the coding efficiency parameter $\alpha_{j}$ and the variance $\sigma j$ for all subbands $j=1,2, \ldots, N$ of the image to compute the assigned bit rate $R i$ for the subband $i$.

\section{Experimental Results}

We carry out computer simulations in Matlab environment, two test images are used in the experiments: Lena (256*256*8bits) and Cameraman (256*256*8bits). A 9/7 filter 
with a three level spatial decomposition is used to calculate the wavelet coefficients. The target bit rate is $0.27 \mathrm{bpp}, 0.45 \mathrm{bpp}, 0.7 \mathrm{bpp}$ for Lena, and $0.28 \mathrm{bpp}, 0.48 \mathrm{bpp}, 0.72 \mathrm{bpp}$ for cameraman, respectively. The image quality is evaluated by using the Peak Signal to Noise Ratio (PSNR). Table 1 gives the reconstruction images comparison for the six cases. From the results, it can be shown that, compared with the scalar quantization using fixed rate distribution strategy, the proposed vector quantization and rate controlling coding system can obviously reduce the bit rate under the same PSNR. It can reduce bit rate at least $2 \%$, and the two of six cases can reduce bit rate more than $10 \%$. Figure 7 shows the reconstruction images comparison of Lena, and Figure 8 shows the reconstruction images comparison of Cameraman, respectively. It evidently shows that the compression ratio and the PSNR are inversely related, i.e., if we use larger codebook size and with more complexity we can achieve higher PSNR, it also shows that, they have good visual quality with adequate bit rate and can be used in most image processing system.

\section{Conclusions}

In this work, we propose wavelet based image coding system using lattice vector quantization and rate controlling. We first present wavelet based image coding system using lattice vector quantization, and then we investigate the rate distortion function for single subband. By Lagrangian optimization algorithm, a rate control scheme is proposed. The experiment results illustrate that the proposed image coding system can improve the coding effect in terms of PSNR.

\section{Table 1. The Reconstruction Images Comparison of Typical Scalar Quantization using Fixed Rate Allocation Scheme and Vector Quantization using Proposed Rate Controlling Scheme}

\begin{tabular}{|c|c|c|c|c|}
\hline Test Image & Scheme & $\begin{array}{c}\text { Bpp } \\
(\text { PSNR:26 })\end{array}$ & $\begin{array}{c}\text { Bpp } \\
(\text { PSNR:29) }\end{array}$ & $\begin{array}{c}\text { Bpp } \\
\text { (PSNR:32) }\end{array}$ \\
\hline \multirow{2}{*}{ Lena } & SQ & 0.3 & 0.5 & 0.82 \\
\cline { 2 - 5 } & VQ & 0.27 & 0.45 & 0.7 \\
\hline Cameraman & SQ & 0.31 & 0.49 & 0.85 \\
\cline { 2 - 5 } & VQ & 0.28 & 0.48 & 0.72 \\
\hline
\end{tabular}



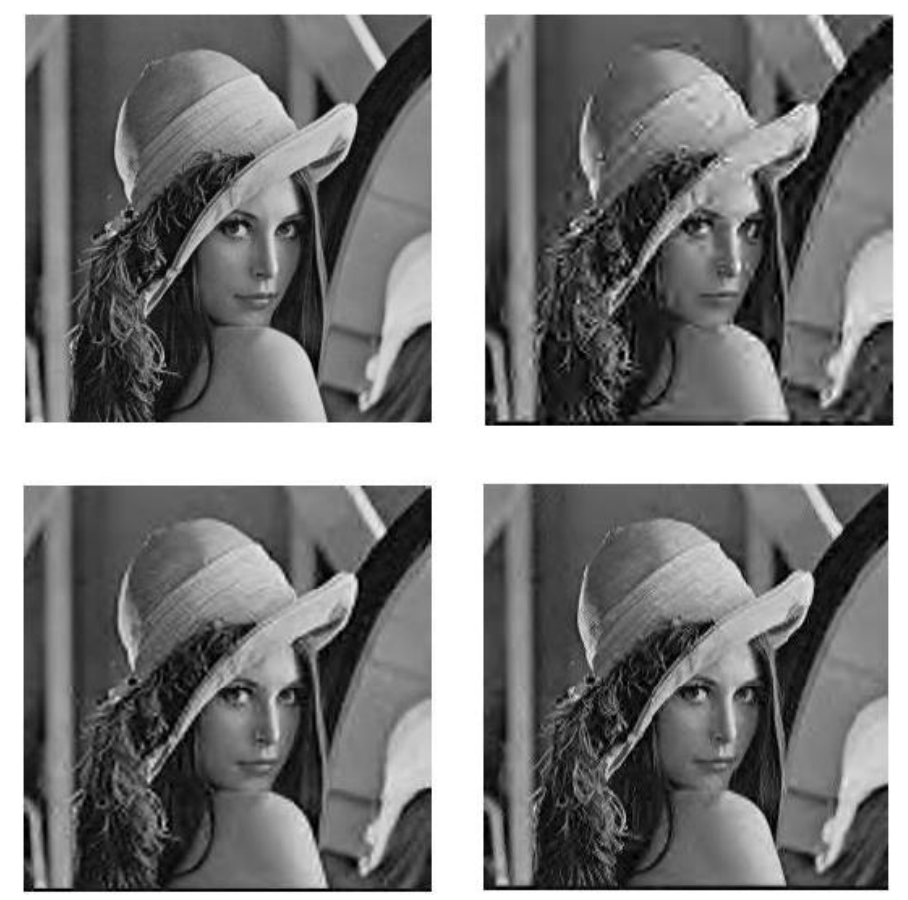

Figure 7. Top Left: Original Lena Image; Top Right: Image using 0.27bpp; Bottom Left: Image using 0.45bpp; Bottom Right: Image using 0. 7bpp
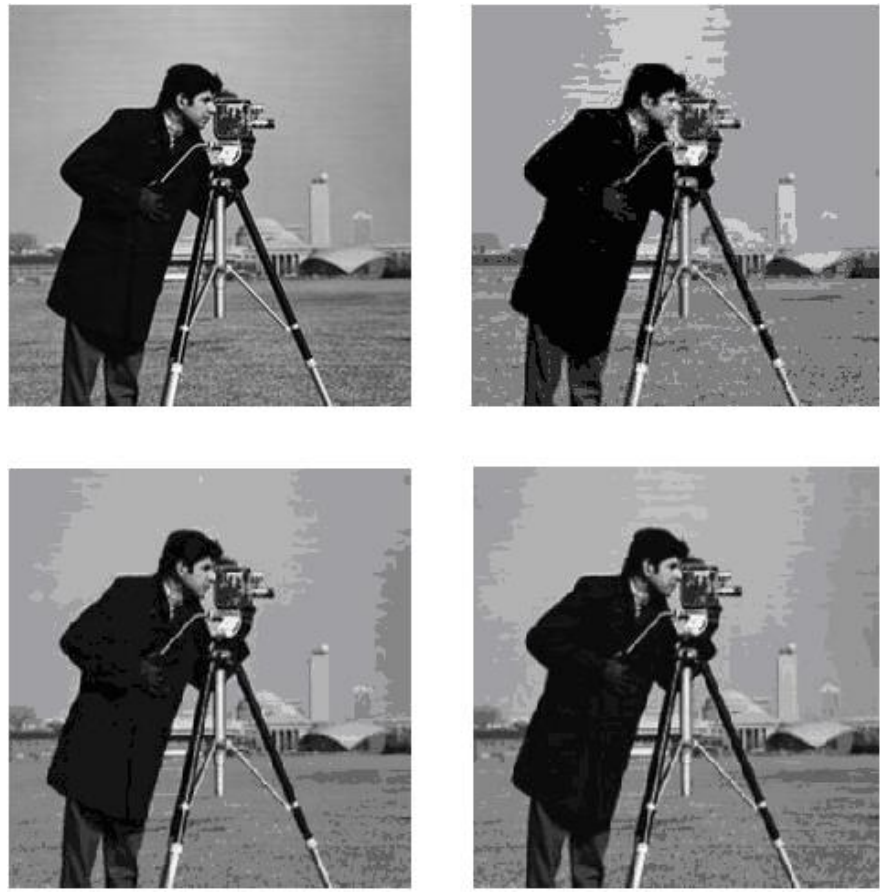

Figure 8. Top Left: Original Cameraman Image; Top Right: Image using 0.28bpp; Bottom Left: Image using 0.48bpp; Bottom Right: Image using 0. $72 \mathrm{bpp}$ 


\section{Acknowledgements}

This work is supported by the Project of Heilongjiang Province Education Department (12511125), Harbin science and technology research special fund project for innovative talents (2012RFQXG090), and the Natural Science Foundation of Heilongjiang Province (F201114). The authors thank Dr Hong Zhu of Yanshan University for her helpful suggestions about the two dimensional wavelet transform of image.

\section{References}

[1] J. M. Shapiro, "Embedded image coding using zerotrees of wavelet coefficients", IEEE Transactions on Signal Processing, vol. 41, no. 12, (1993), pp. 3445-3462.

[2] C. Christopoulos, A. Skodras and T. Ebrahimi, "The JPEG2000 still image coding system: an overview", IEEE Transactions on Consumer Electronics, vol. 46, no. 4, (2000), pp. 1103-1127.

[3] N. Chen and J. Zhu, "Multipurpose Speech Watermarking Based On Multistage Vector Quantization of Linear Prediction Coefficients", The Jamal of China Universities of Posts and Telecommunications, vol. 14, no. 4, (2007), pp. 64-69.

[4] S. W. Teng and G. J. Lu, "Image Indexing and Retrieval Based on Vector Quantization", Pattern Recognition, vol. 40, no. 11, (2007), pp. 3299-3316.

[5] N. Gregory, A. R. Davies and S. Boussakta, "Video Compression Employing Algorithm Using Temporal Blocking Structures", Electronics Letters, vol. 37, no. 9, (2001), pp. 563-564.

[6] A. I. Gonzalez, M. Grana, J. R. Cabello and A. Danjou, "Experimental Results of an Evolution Based adaptation Strategy for VQ Image Filtering”, Information Scienees, vol. 133, (2001), pp. 249-266.

[7] W. Qi, Z. Li, W. Feng, L. Shi-peng and Z. Yu-zhuo, "A Rate Allocation Scheme for Progressive Fine Granular Scalable Video Coding”, ACTA Electronical Sinica, vol. 30, no. 2, (2002) February.

[8] V. Seran and L. P. Kondi, "Quality Variation Control for Three-Dimensional Wavelet-Based Video Coders", EURASIP Journal on Image and Video Processing, (2007).

[9] I. Daubechies, "Ten Lectures on Wavelets", SIAM, New York, USA, (1992).

[10] M. J. Wainwright, E. P. Simoncelli and A. S. Willsky, "Random cascades on wavelet trees and their use in modeling and analyzing natural imagery”, Applied Comput. Harmon. Anal., vol. 11, (2001), pp. 89-123.

[11] T. Voinson, L Guillemot and J. M. Moureaux, "Image compression using Lattice Vector Quantization with code book shape adapted thresholding", IEEE International Conference on Image Processing, Rochester, New York, (2002) September.

[12] L. Guillemot, Y. Gaudeau and J. M. Moureaux, "A new fast bit allocation procedure for image coding based on wavelet transform and dead zone lattice vector quantization”, IEEE ICIP, Genoa, Italy, (2005), September $10-15$.

[13] J. M. Moureaux, P. Loyer and M. Antonini, "Low complexity indexing method for Zn and Dn lattice quantizers”, IEEE Trans. Commun., vol. 46, no. 12, (1998) December, pp. 1602-1609.

[14] T. M. Cover and J. A. Thomas "Elements of Information Theory", New York, NY: John Wiley and Sons, Inc, (1991).

[15] M. Dai, D. Loguinov and H. Radha, "Rate-Distortion Modeling of Scalable Video Codes", IEEE ICIP, Singapore, (2004) October 24-27.

\section{Authors}

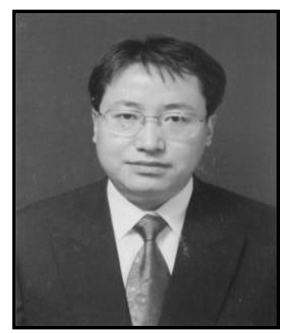

Lizhi Zhang, was born in Heilongjiang, China, in 1968. He received the Master degree in communication engineering from Harbin Institute of Technology, Harbin, China, in 1995.

He joined Harbin University of Commerce in 2004. His research areas are image coding, vector quantization, and information theory. 


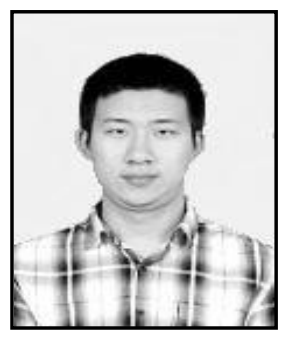

Mingrui Zhang, was born in Heilongjiang, China, in 1989. He received the Bachelor degree in electronic information engineering from Harbin Institute of Technology at Weihai, Weihai, China, in 2012.

He joined Harbin Engineering University in 2012, and he is now a graduate student. His research interests include image coding, information theory, and sensor design.

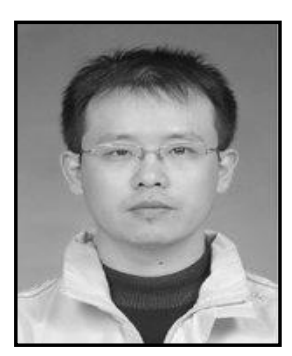

Qinghe Pan, was born in Heilongiiang, China, in 1981. He received Ph.D. from Harbin Institute of Technology, Harbin, China, in 2011.

He joined Harbin University of Commerce in 2011. His research interests include image coding, information theory, and computer vision. 
International Journal of Signal Processing, Image Processing and Pattern Recognition Vol.7, No.2 (2014) 